\title{
THE 2-GENERALIZED KNOT GROUP DETERMINES THE KNOT
}

\author{
SAM NELSON AND WALTER D. NEUMANN
}

To the memory of Xiao-Song Lin

\begin{abstract}
Generalized knot groups $G_{n}(K)$ were introduced independently by Kelly (1991) and Wada (1992). We prove that $G_{2}(K)$ determines the unoriented knot type and sketch a proof of the same for $G_{n}(K)$ for $n>2$.
\end{abstract}

\section{The 2-Generalized KNOT GROUP}

Generalized knot groups were introduced independently by Kelly [5] and Wada [10]. Wada arrived at these group invariants of knots by searching for homomorphisms of the braid group $B_{n}$ into $\operatorname{Aut}\left(F_{n}\right)$, while Kelly's work was related to knot racks or quandles $[1,4]$ and Wirtinger-type presentations.

The Wirtinger presentation of a knot group expresses the group by generators $x_{1}, \ldots, x_{k}$ and relators $r_{1}, \ldots, r_{k-1}$, in which each $r_{i}$ has the form

$$
x_{j}^{ \pm 1} x_{i} x_{j}^{\mp 1} x_{i+1}^{-1}
$$

for some map $i \mapsto j$ of $\{1, \ldots, k\}$ to itself and map $\{1, \ldots, k\} \rightarrow\{ \pm 1\}$. The group $G_{n}(K)$ is defined by replacing each $r_{i}$ by

$$
x_{j}^{ \pm n} x_{i} x_{j}^{\mp n} x_{i+1}^{-1} .
$$

In particular, $G_{1}(K)$ is the usual knot group.

In [9], responding to a preprint of Xiao-Song Lin and the first author [6], Tuffley showed that $G_{n}(K)$ distinguishes the square and granny knots. $G_{n}(K)$ cannot distinguish a knot from its mirror image. But $G_{2}(K)$ is, in fact, a complete unoriented knot invariant.

Theorem 1.1. The 2-generalized knot group $G_{2}(K)$ determines the knot $K$ up to reflection.

We will assume $K$ is a non-trivial knot in the following proof, although it is not essential. It is clear from the proof that the trivial knot is the only knot with $G_{2}(K)=\mathbb{Z}$.

Wada described $G_{n}(K)$ as the fundamental group of the space $M_{n}(K)$ obtained by gluing the boundary torus of the knot exterior to another torus by the map $S^{1} \times S^{1} \rightarrow S^{1} \times S^{1}$ defined by $f\left(z_{1}, z_{2}\right)=\left(z_{1}^{n}, z_{2}\right)$, where $z_{1}$ represents the meridian and $z_{2}$ represents a longitude. We will use this description. We will call the glued-on torus the core torus.

Note that $M_{2}(K)$ is a closed manifold: it can be described as the result of gluing $M b \times S^{1}$ into the knot exterior, where $M b$ denotes the Möbius band. It is clearly Haken, since its fundamental group has a $\mathbb{Z}$ quotient, and it is irreducible and $\mathbb{P}^{2}$-irreducible since its orientation cover is the double of the knot exterior 
and hence irreducible. It therefore follows by Heil's non-orientable extension [3] of Waldhausen's theorem [10] that $M_{2}(K)$ is determined by its fundamental group $G_{2}(K)$.

The core torus $T$ is the product $S^{1} \times S^{1}$ in $M b \times S^{1} \subset M_{2}(K)$, where the first $S^{1}$ is the central circle of the Möbius band. If one cuts $M_{2}(K)$ along the core torus $T$ one recovers the knot complement, from which the knot itself can be recovered by Gordon and Luecke [2]. Thus the theorem follows from the following lemma.

Lemma 1.2. The core torus $T \subset M_{2}(K)$ is, up to isotopy, the unique one-sided torus in $M_{2}(K)$.

Proof. We will use the "geometric version" of the JSJ decomposition. This is described, for instance, in [7, Section 4], but only for orientable manifolds, so we will discuss the non-orientable case briefly here. If the reader prefers to avoid JSJ for non-orientable manifolds (s)he can easily mirror our argument in the orientation cover of $M$.

We restrict to the special case of an irreducible and $\mathbb{P}^{2}$-irreducible manifold $M$ whose boundary components are tori or Klein bottles. The JSJ decomposition (geometric version $^{1}$ ) then decomposes $M$ along an embedded closed surface and is characterized by the first three of the following properties.

(1) The surface is a disjoint union of essential tori and Klein bottles,

(2) $M$ is decomposed into simple (i.e., essential tori and annuli are boundary parallel) and Seifert fibered pieces, with no piece being an interval bundle over a torus or Klein bottle.

(3) The surface is minimal in the sense that it is, up to isotopy, a subset of any other surface with the above properties.

(4) Any essential embedded torus or Klein bottle in $M$ can be isotoped to be a component of the JSJ surface, to lie in a neighborhood of one of its components, or to lie in a Seifert fibered piece of the decomposition.

A short geometric proof of the existence and uniqueness of this decomposition was given in [7] in the orientable case and can easily be extended to the nonorientable case. Alternatively, one decomposes the orientation cover of $M$ (or any other orientable finite cover) and then uses the naturalness of the geometric JSJ decomposition to descend to $M$. One of the features of the geometric version of JSJ is that it lifts correctly in finite covers, and using standard minimal surface technology one can isotop it to be preserved by any finite group action.

Consider now the union of the JSJ surface $F$ for the knot exterior and the core torus $T$. This is a surface that satisfies conditions (1) and (2) so it contains the JSJ surface. It follows easily that the JSJ surface is either $F$ or $F \cup T$. In the latter case we know that any essential torus other than $T$ is isotopic into the complement of $T$, hence embeds in $S^{3}$, and is thus two-sided, so $T$ is the only onesided torus. So assume the JSJ surface is $F$. This only happens if the piece of the JSJ decomposition of the knot exterior that contains the boundary is itself Seifert fibered, so $K$ is either

- a $(p, q)$ torus knot for some $1<q<p$ (and $F$ is empty) or

- a sum of $k>1$ prime knots.

\footnotetext{
${ }^{1}$ For manifolds covered by a torus bundle over the circle the JSJ decomposition described here is not necessarily the geometric one (the geometric JSJ decomposition is trivial in this case, overlooked in section 4 of [7]). It is an exercise to see that $M_{2}(K)$ is never of this form.
} 
In the first case $M$ is Seifert fibered over the orbifold $P(p, q)$ which is $\mathbb{P}^{2}$ with two orbifold points of degrees $p$ and $q$ respectively. In the second case the Seifert fibered component containing $T$ fibers over an orbifold $Q(k)$ which is $k$-holed disk with one boundary being a mirror boundary (the image of $T$ ).

Any essential surface in a Seifert fibered manifold is isotopic to a vertical surface (union of fibers) or a transverse one (transverse to all fibers). A transverse surface could only be closed in the first case, but it is then hyperbolic since it covers the base orbifold $P(p, q)$ which is hyperbolic. Thus in each case an essential embedded torus must be vertical. In the first case, if it is one-sided it must lie over an orientation reversing closed loop in $P(p, q)$, and there is just one such loop up to isotopy (avoiding the orbifold points), and it gives the torus $T$. In the second case an essential torus other than $T$ is the inverse image of a closed loop that does not meet the mirror boundary or of a connected 1-orbifold (i.e., an arc) with both ends on the mirror boundary, and any such torus is two-sided.

\section{THE $n$-GENERALIZED KNOT GROUP}

The result holds also for $G_{n}(K)$ for $n>2$. Here is an outline of the argument.

In this case $M_{n}(K)$ is not a manifold, so we cannot use 3-manifold JSJ. Instead we work directly with the group $G_{n}(K)$, using the Scott-Swarup version of JSJ for groups [8]. For $n>3$ Scott-Swarup JSJ decomposes $M_{n}(K)$ as a graph of groups corresponding to the JSJ decomposition of the knot exterior (in a version close to classical JSJ rather than geometric JSJ), together with an additional edge and vertex (of type " $V_{0}$ " in the terminology of SS-JSJ) as follows: the edge group and vertex group are the peripheral subgroup of the knot exterior and $\pi_{1}(T)$. The edge is characterized as the only edge of the graph of groups whose group injects with finite index in a vertex group. The knot group can thus be recovered as the fundamental group of the graph of groups which results by removing this edge and its end vertex. Also, the peripheral subgroup of the knot group is recovered as the edge group for this edge. Finally, the knot is determined by knot group plus peripheral subgroup by Gordon and Luecke [2]. For $n=3$ (and 2) one can use essentially the same argument, but there is an extra $V_{0}$-vertex corresponding to the peripheral $\mathbb{Z} \times \mathbb{Z}$ of the knot group, and the vertex for $\pi_{1}(T)$ is a $V_{1}$-vertex.

$G_{n}(K)$ is also defined for links, but is not a complete invariant. Since it can be functorially derived from the rack (or quandle) of the link, it cannot determine more than the rack determines (see [1]). What $G_{n}(K)$ determines for a decomposable link is the exteriors of the indecomposable sublinks, but since they are recovered without knowledge of their orientation, one cannot reassemble the whole link exterior. Moreover, since $G_{n}(K)$ (unlike the rack) does not know the peripheral structure (i.e., the elements given by meridians), it cannot necessarily recover an indecomposable link (but Chris Tuffley pointed out to us that if one knows $G_{n}(K)$ for infinitely many $n$ then one does know meridians up to sign).

Acknowledgements. The second author acknowledges NSF support under grant DMS-0456227. The authors thank Peter Scott, Colin Rourke and Christopher Tuffley for useful correspondence. This paper was born when the authors became acquainted on flight AA3394 (Baton Rouge to Dallas 30/Mar/08), where they would not have met but for Xiao-Song Lin, to whose memory they dedicate this paper. 


\section{REFERENCES}

[1] R. Fenn and C. Rourke, Racks and links in codimension two. J. Knot Theory Ramifications 1 (1992) 343-406.

[2] C. Gordon and J. Luecke, Knots are determined by their complements. J. Amer. Math. Soc. 2 (1989), 371-415.

[3] W. Heil, On $P^{2}$-irreducible 3-manifolds. Bull. Amer. Math. Soc. 75 (1969), 772-775.

[4] D. Joyce. A classifying invariant of knots, the knot quandle. J. Pure Appl. Algebra 23 (1982) 37-65.

[5] A. J. Kelly. Groups from Link Diagrams. Doctoral Thesis, U. Warwick (1990).

[6] X.-S. Lin and S. Nelson, On Generalized Knot Groups, arXiv:math.GT/0407050

[7] W.D. Neumann and G. Swarup, Canonical decompositions of 3-manifolds. Geom. Topol. 1 (1997), 21-40

[8] P. Scott and G. Swarup, Regular neighbourhoods and canonical decompositions for groups. Astérisque 289 (2003), vi+233 pp.

[9] C. Tuffley, Generalised knot groups distinguish the square and granny knots, arXiv:0706.1807

[10] M. Wada. Group invariants of links. Topology 31(1992) 399-406.

[11] F. Waldhausen, On irreducible 3-manifolds which are sufficiently large. Ann. of Math. 87 (1968), 56-88.

Department of Mathematics, Pomona College, 610 N. College Avenue, Claremont, CA 91711

E-mail address: knots@esotericka.org

Department of Mathematics, Barnard College, Columbia University, New York, Ny 10027

E-mail address: neumann@math.columbia.edu 